\title{
Transferrin microheterogeneity as a probe in normal and disease states
}

\author{
G. DE JONG ${ }^{1,2}$, R. FEELDERS ${ }^{1}$ W. L. VAN NOORT ${ }^{1}$ and H. G. VAN EIJK ${ }^{1 *}$ \\ 'Department of Chemical Pathology, Medical Faculty, Erasmus University, 3000 DR Rotterdam, The Netherlands \\ ${ }^{2}$ Department of Internal Medicine II, Academic Hospital Dijkzigt Rotterdam, Dr Molewaterplein 40, 3000 DR \\ Rotterdam, The Netherlands
}

Received 11 July 1994

\begin{abstract}
Isoelectric focusing of iron saturated serum has been established as a convenient method for showing transferrin glycan microheterogeneity. In a clinical setting, the method is used in the detection of cerebrospinal fuid leakage, the screening for surreptitious alcohol abuse and in the ditgnosis of the carbohydrate deficient glycoprotein syndrome. In normal physiological states it can also be used as a tool to probe for changes in N-glycosylation.
\end{abstract}

Keywords: transferrin microheterogeneity, isoelectric focusing, iron saturated serum, cerebrospinal fluid leakage, alcohol abuse, carbohydrate deficient glycoprotein syndrome, $\mathrm{N}$-glycosylation

\section{Introduction}

Microheterogeneity is defined in this article as the structural variation in monomeric proteins due to differences in $\mathrm{N}$-linked glycan structure. Many methods are now available to measure structural diversity but most often these only partially describe the situation. Although exact description of glycan structure is possible, this is impractical in view of the relatively large amount of protein needed in such an analysis.

Transferrin (Tf) is the iron carrying monomeric glycoprotein of vertebrates, and plays an important physiological role in iron homeostasis, regulation of growth and non-immunological defence $[1,2]$. It exists in many different forms which are determined by genetic polymorphism, iron saturation and glycan composition. These factors affect the electrophoretic behaviour of Tf. The most common phenotype (more than 95\% in European populations) has been designated TfC, whereas the anodal and cathodal forms are designated TfB and TfD respectively. Phenotypes within the main groups are given subscripts ( $\mathrm{TfD}_{\mathrm{Ch}}, \mathrm{TfD}_{1}$, etc). The aminoacid changes in some of these variants have been shown to be a single aminoacid substitution [3]. Until 1978, TfC was considered to be a single variant. However, using isoelectric focusing (IF), TfC was shown to consist of many subtypes; the total number of $\mathrm{C}$-variants is now claimed to be $16[4,5]$. The degree of $\mathrm{Tf}$

${ }^{*}$ To whom correspondence should be addressed polymorphism in most populations has, therefore, spuriously increased to about $29 \%$. Although distributions in different
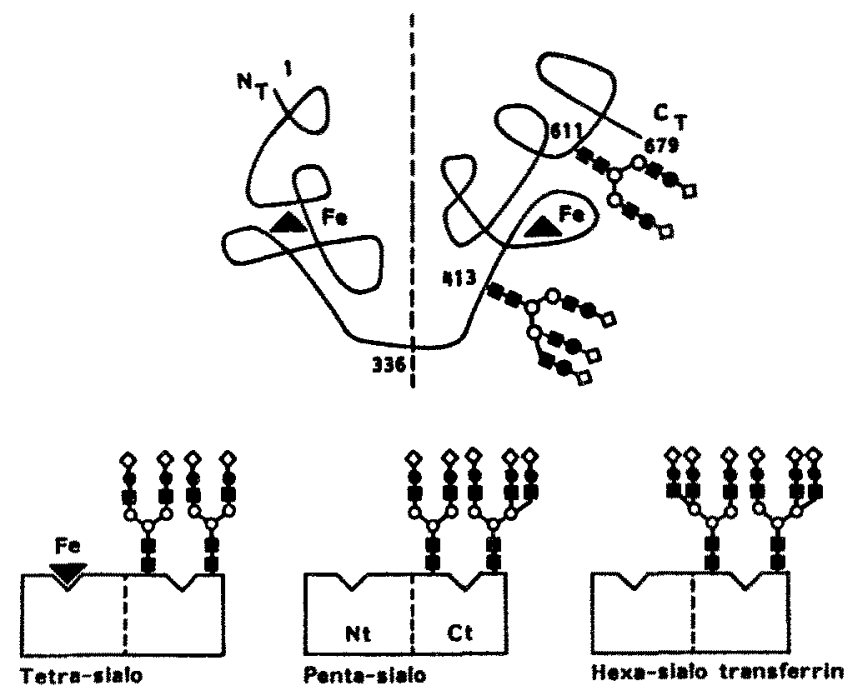

Legend:

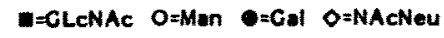

Figure 1. Schematic representation of the transferrin molecule carrying two $\mathrm{N}$-linked glycans in the $\mathrm{C}$-terminal domain. Three microheterogeneous forms of human transferrin are shown at the bottom of the figure. Taken with permission from reference 2 . 




Figure 2. Concanavalin A Sepharose chromatography of human serum transferrin.

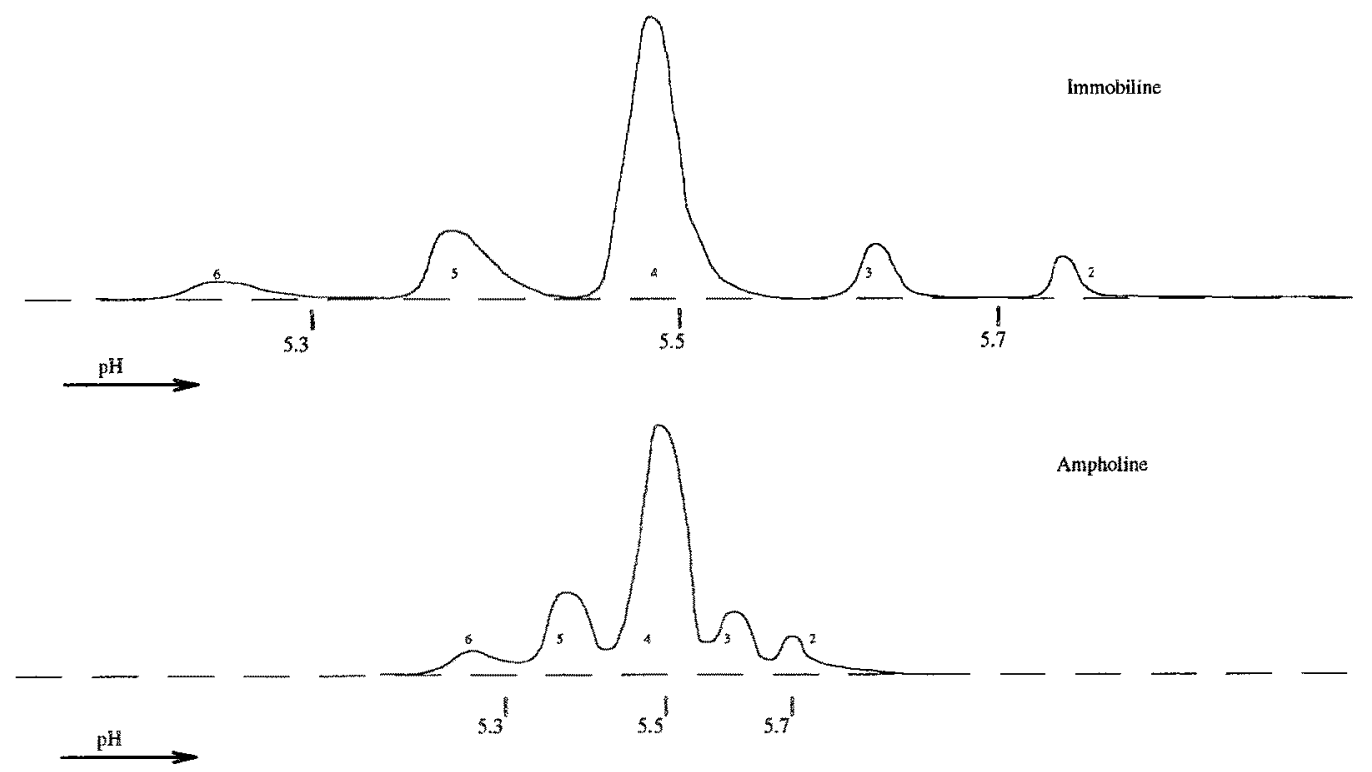

Figure 3. Top: densitometric scan of sialotransferrins separated on immobilines. Bottom: separation in ampholine gel. Cathode at the right hand side. Numerals indicate number of sialic acid residues per molecule.

populations vary, of all 38 or more variants only four occur with a frequency of over $1 \%$ [5].

Amongst the serum glycoproteins, glycosylation of $\mathrm{Tf}$ is relatively simple (Fig. 1). Most other glyoproteins contain three or more $\mathrm{N}$-linked glycans and some O-linked glycans. Tf contains no O-linked glycans and only two $\mathrm{N}$-linked complex type glycans $[6,7]$. This means that the electrophoretic pattern for $\mathrm{Tf}$ is relatively simple. Similarly, Concanavalin A affinity chromatography yields a limited number of well separated fractions which are easily identified (Fig. 2).
IF using immobilines is one of the most sensitive separation methods available and separates molecules on the basis of differences in ionizable groups. Immobilines are used to create a fixed $\mathrm{pH}$-gradient in a gel. Proteins which are added to the gel move between cathode and anode until they reach a position in the gel where the $\mathrm{pH}$ is equal to their isoelectric point. This causes the protein to accumulate at this position in the gel. Hence, accurate and reproducible quantification of Tf variants is possible.

Initially, quantification of $\mathrm{Tf}$ was performed by crossed (rocket) immunoelectrophoresis. The area under the Tf 
precipitation peaks was measured and the relative amounts of each fraction was determined by determining the $\%$ area in each fraction. In this article relative amounts in each fraction have been quantified by scanning the gel using a laser densitometer after immunofixation and subsequent staining. This gives numerically identical results, but is much less labourintensive and gives better base line separation and reproducibility. In Fig. 3 typical results are shown, emphasizing the superior separation for $\mathrm{Tf}$ in an immobiline gel compared with that obtained in an ampholine gel.

In this article, current knowledge on the investigation of $\mathrm{N}$ linked glycan heterogeneity of Tf is summarized, and the use of IF of iron saturated Tf in serum as a tool to detect alterations in $\mathrm{N}$-glycosylation in pathophysiological situations is highlighted.

\section{Materials and methods}

\section{Sample treatment}

Venous blood samples were obtained between 10.00 am and $12.00 \mathrm{am}$ in glass tubes without anticoagulant. Omitting the anticoagulant is a relevant part of the procedure, as we and others have found that this may influence the IF [8]. After clot retraction, the sample was centrifuged for $20 \mathrm{~min}$ at $1200 \times \mathbf{g}$ and $4{ }^{\circ} \mathrm{C}$. The serum was used immediately or stored at $-20^{\circ} \mathrm{C}$, or $-70^{\circ} \mathrm{C}$ until it was used. Before storage or application to the gels, complete iron saturation of the $\mathrm{Tf}$ was ensured by mixing $100 \mu \mathrm{l}$ of serum with $5 \mu \mathrm{l}$ of $0.5 \mathrm{~mol}^{-1} \mathrm{NaHCO}_{3}$ and $3 \mu \mathrm{l}$ of $10 \mu \mathrm{mol}^{-1} \mathrm{Fe}$ (III) citrate and standing at room temperature for $1 \mathrm{~h}$. Rabbit anti human Tf anti-sera was made in the laboratory compared favourably with commercially-available anti sera (Dako-Denmark).

\section{Analytical isoelectric focusing and crossed-immunoelectro- phoresis}

This method involved two steps; the first, separated the proteins and the second, identified and quantified the separated components.

The Tf subfractions were separated on polyacrylamide gelstrips in an immobilized pH-gradient by IF. Strips were cut from an Immobiline Dry Plate, $\mathrm{pH} 4.9$ - 6.1 (Pharmacia Biotech, Sweden), which had previously been rehydrated in aqua bidest to a final weight of $20 \mathrm{~g}$ per gel and placed on a thermostatically-controlled baseplate of an IF apparatus kept at $10^{\circ} \mathrm{C}$. Anode and cathode strips soaked in $10 \mathrm{mmol} \mathrm{l}^{-1}$ glutamic acid and $10 \mathrm{mmol} \mathrm{l}^{-1} \mathrm{NaOH}$, respectively, were placed over the ends of the Immobiline strips. An aliquot containing $2 \mu 1$ of an iron-saturated serum (diluted 1:10) was applied at the cathodal side of the strips and a voltage of $1 \mathrm{kV}$ was applied for $18 \mathrm{~h}$. After IF, strips were covered with $50 \mu \mathrm{l}$ rabbit anti human Tf antibody (purchased from Dako, Copenhagen, Denmark, titre 1:2800 or an in-house prepared antibody). Dilution of the sample prior to IF was necessary because it was found that antigen excess could cause predominant subfractions to dissolve during the overnight saline wash following the antibody treatment. Immunoprecipitated bands were stained in a solution of $12 \%(\mathrm{w} / \mathrm{v})$ trichloroacetic acid containing $0.1 \%$ Coomassie Brilliant Blue G-250, and rinsed with demineralized water and air dried.

Tf bands were quantified with the Ultroscan XLaser Densitometer. Results were processed using the Gelscan XL (2.1) software (Pharmacia Biotech).

\section{Tf concentrations}

TF concentrations were measured turbidimetrically [9] using a Boehringer antibody ES22. The Tf immunoprecipitated with sheep-anti-human-Tf in an $0.1 \mathrm{~mol} \mathrm{1}^{-1}$ $\mathrm{Na}_{2} \mathrm{HPO}_{4} / \mathrm{KH}_{2} \mathrm{PO}_{4}$ buffer $\mathrm{pH} \quad 7.5$, containing $4 \%$ polythyleneglycol.

\section{Concanavalin A affinity chromatography}

Tf isolated from serum by immunoaffinity chromatography was separated into the following three fractions: nonbinding, weakly binding and strongly binding using the procedure described by Hatton and Berry [10]. Diferric Tf was dialysed against $0.05 \mathrm{~mol} \mathrm{l}^{-1}$ Tris $/ \mathrm{HCl}, \mathrm{pH}=7.6$ containing $1 \mathrm{~mol} \mathrm{l}^{-1}$ $\mathrm{NaCl}, 1 \mathrm{mmol} \mathrm{l}^{-1} \mathrm{CaCl}_{2}, 1 \mathrm{mmol}{ }^{-1} \mathrm{MnCl}_{2}, 1 \mathrm{mmol}^{-1} \mathrm{MgCl}_{2}$, and approximately $1 \mathrm{mg}$ Tf per $\mathrm{g}$ wet gel was loaded on to a Sepharose concanavalin A (Con A) column. The column was pre-equilibrated and eluted with the above buffer. Fractions of $5 \mathrm{ml}$ were collected and most of the Tf (about $76 \%$ ) was eluted after adding $\alpha$-methyl-glucopyranosyl $\left(0.15 \mathrm{~mol} \mathrm{l}^{-1}\right)$ to the buffer. The nonbound and the weakly bound fractions amounted to approximately $4 \%$ and $20 \%$ respectively.

\section{Results and discussion}

For the interpretation of IF patterns, detailed knowledge of glycan synthesis and metabolism is required. For reviews on this topic see [11-15]. Evolutionarily, glycan synthesis is one step up the ladder compared to protein synthesis, particularly in its coding mechanism. Through branching, different anomeric configurations and types of bonding, a vast combination of structural classes are created. However, glycans are distinct from polypeptides and nucleic acids, in the relatively small molecular size at which this diversity is attained. They are also distinct from nucleic acids and polypeptides in that they are not coded for by an accurate template mechanism. Essentially, glycan structure is coded for by the intrinsic information present in the primary, secondary and tertiary structure of the polypeptide core, in conjunction with characteristics of the enzymes involved in the synthetic machinery; as well as any interaction with the matrix in which the glycan is processed. Consequently, the outcome is not easily defined, and the final product is very microheterogeneous.

With differic Tf the picture is simpler because the heterogeneity visualized through variations in the isoelectric point in a sample is a reflection of the number of sialic acid residues per molecule. In complex $\mathrm{N}$-glycans, the degree of branching determines this number. Further variation can be 
Table 1. Transferrin microheterogeneity in normal serum as distinguished by degree of branching of glycans (Con A separation) and by number of sialic acids per molecule (IF separation expressed as a \%).

\begin{tabular}{|c|c|c|c|c|}
\hline & \multicolumn{3}{|c|}{ Concanavalin A separation } & \multirow{2}{*}{$\frac{\text { IF separation }}{\text { Total }(\%)}$} \\
\hline & bi-bi-antennary & bi-tri-antennary & tri-tri-antennary & \\
\hline \multicolumn{5}{|c|}{ Sialotransferrin } \\
\hline \multicolumn{5}{|c|}{ Fractions } \\
\hline 0 & - & - & - & - \\
\hline 1 & - & - & - & - \\
\hline 2 & 2.3 & 0.2 & - & 2.5 \\
\hline 3 & 7.1 & 0.2 & - & 7.3 \\
\hline 4 & 65.4 & 0.8 & 0.1 & 66.3 \\
\hline 5 & 0.7 & 18.0 & 0.2 & 18.9 \\
\hline 6 & - & 1.1 & 3.7 & 4.8 \\
\hline 7 & - & - & 0.1 & 0.1 \\
\hline 8 & - & - & - & - \\
\hline Total & 75.5 & 20.3 & 4.1 & 99.9 \\
\hline
\end{tabular}



Figure 4. Densitometric scans of transferrin microheterogeneity in the course of acute hepatitis. Cathode at the right hand side of the figure. (A) Normal scan. (B) Isoelectric focusing pattern at presentation with severe hepatitis. (C) Pattern in the convalescent period. Gradual normalization of the pattern coincided with a decrease in disease activity.

present as a result of either aberrant or incomplete synthesis or desialylation. To assess the influence of the latter two factors, an IF analysis was performed on the three Tf fractions that can be separated by Con A affinity chromatography.

Eighteen Tf components were detected by IF in the Con A fractions (Table 1). Only 13 are shown in the Table because only trace quantities of the 0,1 and 8 sialotransferrins were visible on the gel. The Table shows that the different forms of Tf [4-6] after IF can be used to predict the type of complex glycan that is present. Therefore, by virtue of its comparatively simpler carbohydrate structure, we have used IF of Tf as a probe to monitor changes in protein glycosylation in physiological and pathological states. 
With respect to the catabolism of glycoprotein glycans in the circulation; it is our experience that in normal healthy individuals little variation occurs as a result of this type of modification. Tf can be stored at $4^{\circ} \mathrm{C}$ for long intervals without significant changes in the distribution of microheterogeneous forms. On the other hand, in a number of disease states such as acute hepatitis and uraemic haemolytic syndrome, and following pneumococcal sepsis, we have observed a very pronounced shift in the pattern towards forms with less than the usual number of sialic acids (Fig 4). As these changes were reversible, with the pattern gradually normalizing with the reduction in disease activity, we believe this to be the result of enzymes released into the circulation through cell lysis related to the disease process, possibly augmented by bacterial and/or viral enzymes. Between and within individuals the variation in Tf microheterogeneity as assessed by IF is minimal [16]. Also, in all groups with an established disease or physiological state the pattern of distribution is remarkable in its uniformity [16]. Variation in microheterogeneity is, therefore, not just the result of random variations. The consistency of $\mathrm{Tf}, \mathrm{IF}$ patterns is characteristics and demonstrates that Tf glycosylation must be closely controlled, although the mechanism for this is unknown. Thus, IF of serum Tf can be used to assess the control of glycosylation.

Different degrees of sialylation and galactosylation of human Tf in cerebrospinal fluid (CF), amniotic fluid and synovial fluid have been described $[17,18]$. The occurrence of a particularly high proportion of asialo Tf in CF has been put to clinical use. Early diagnosis of CF leakage, most commonly presenting as otorrhoea or rhinorrhoea, can be of vital importance in recurrent meningitis. Cerebrospinal otorrhoea and rhinorrhoea are notoriously difficult to diagnose, often requiring labour-intensive, costly, radiographic procedures that are not without risk to the patient. By analysing the microheterogeneity of the Tf pattern of suspect CF and demonstration of the existence of a pronounced asialo- $\mathrm{Tr}$ band, a diagnosis of $\mathrm{CF}$ leakage can be made [19]. Alternatively, demonstration of the asialo-Tf is also possible by more conventional electrophoretic techniques (in these techniques asialo-Tf is conventionally referred to as the tau-band), but the advantages of the IF approach are higher specificity and, in particular, higher sensitivity.

Variation in the microheterogeneity pattern of $\mathrm{Tf}$ can also be found or induced by several pathological and physiological conditions (Fig. 5). A highly aberrant IF pattern has been described in children suffering from a rare syndrome dominated by neurological abnormalities $[20,21]$. The di- and asialofractions are present in high concentrations, a phenomenon which also occurs in the CF of these children. Tf is not the only aberrantly glycosylated protein in this condition. Other glycoproteins such as $\alpha_{1}$ antitrypsin and ceruloplasmin have also been found to be glycosylated differently $[22,23]$. The metabolic error in protein glycosylation in this disease is not well-defined and it is known as the carbohydrate deficient glycoprotein syndrome (CDG). After its first description by



Figure 5. Isoelectric focusing patterns of iron saturated serum after immunofixation with antitransferrin and Coomassie Blue staining. Cathode at top. Lanes 4, 8 and 12: normal sera. Lanes 1, 2, 3 and 7: Carbohydrate Deficient Glycoprotein Syndrome. Lane 9: serum from a patient with high serum sialidase activity due to pneumococcal sepsis. Lane 10: alcoholic. Lane 11: Hodgkin's Disease. Lanes 5 and 6: duplicated normal pattern due to genetic heterozygosity; both $\mathrm{TfC}_{1}$ - and TfD-variant-tetrasialotransferrins are prominent, and other extra intense signal due to pentasialo-TfD and trisialo-Tf, focusing at the same position.

Jaeken et al. [20], additional variants were recognized, so that currently, a further division of the disease into subtypes termed, CDG-I CDG-II, etc. has been made. The exact biochemical defects distinguishing these subtypes are not known and the distinction between them is based on the microheterogeneity pattern of $\mathrm{Tf}$, which in this context acts as an electrophoretic fingerprint.

Several groups have shown that one or more Tf bands detected by IF are more pronounced in the sera of alcoholics. This is the reflection of an increased concentration of molecules with a low sialic acid content. Detection of these bands can be used as a clinical test for surreptitious alcohol abuse. The specificity of such a test is inadequate, however, since organic solvent intoxication can also cause the increased occurrence of $\mathrm{Tf}$ variants with lower sialic content.

More subtle changes are induced in pregnancy, rheumatoid arthritis (RA) and idiopathic haemochromatosis $[16,24,25]$. Besides confirming the recognized effect of pregnancy on total Tf synthesis, we have also shown a consistent and progressive redirection of synthesis towards highly sialylated Tfs in the course of pregnancy [24]. This effect became statistically significant in the second trimester, and the trend appeared to be continued up to the last week of pregnancy. Compared to any of the other investigated groups, pregnancy had the most pronounced effect on the microheterogeneity of Tf. After delivery, these changes are rapidly reversed. This is demonstrable as early as 4 days after delivery, and 5 weeks later both the concentrations and the subfraction-pattern are virtually normal (Fig. 6). Women using oral the contraceptives 




Transferrin fraction concentrations ( $\mathrm{mcmol} / \mathrm{l})$

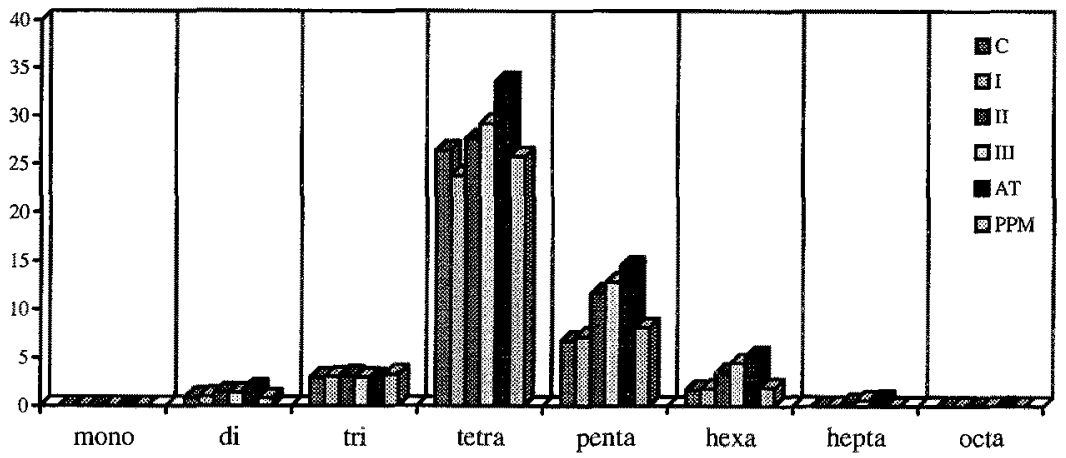

Figure 6. Transferrin microheterogeneity in the course of pregnancy. Sialotransferrin fractions are indicated on X-axis. C: Control females, I, II, III: 1st, 2nd and 3rd trimester of pregnancy respectively, AT: full term, PPM 1 month post partum. Top: proportional distribution of sialotransferrin fractions $(\%)$ and in lower half corresponding concentrations $\left(\mu \mathrm{mol} 1^{-1}\right)$.

displayed a similar trend: the concentration of total Tf was increased, and compared with the controls, more of the increase was accounted for by the highly sialylated variants. Compared to pregnancy, the pattern differed in that at the same concentration of total $\mathrm{Tf}$, the change in proportion was less marked than in pregnancy.

Iron deficiency is accompanied by an increased total $\mathrm{Tf}$, the group average rising by $48.2 \%$. Relative proportions are shifted considerably less towards the highly sialylated fractions, reaching statistical significance only for the penta-sialo Tf. Compared to pregnancy, total Tf concentration in iron deficiency is higher, and most of the actual increment in concentration is due to increased production of tetrasialo-Tf.

RA patients without anaemia have been compared with RA patients with iron deficiency, and RA patients with anaemia of chronic diseases (ACD). Patients with active RA generally have a lower total $\mathrm{Tf}$ concentration. The decrease is less in the subgroup with iron deficiency, but still significantly lower when compared to the healthy controls. The microheterogeneity pattern is again changed in favour of the highly sialylated Tfs. This change is less pronounced in the non-anaemic RA patients and most pronounced in the ACD-group, with the iron deficiency group occupying the intermediate position. In both the iron deficiency and the ACD-subgroups, the concentrations of penta-, hexa- and heptasialo $\mathrm{Tfs}$ are not significantly depressed compared to controls, while the total Tf concentration in these groups is diminished by $15.1 \%$ and $38.8 \%$ respectively. The tetrasialo $\mathrm{Tf}$ is significantly depressed in both proportion and in concentration in all RA subgroups, and a similar effect is observed for the di- and trisialo-Tf (Fig. 7).

It is possible to draw certain conclusions on the coupling between protein and glycan synthesis from our results for different physiological and pathological states. Results on sera from pregnant women indicate that protein core synthesis and changes in glycosylation towards higher branched glycans are not linked, since the changes in the forms with tri- and tetraantennary chains correlated better with duration of gestation than with the concentration of total Tf [16]. Also, women on oral contraceptives showed less change in microheterogeneity when compared to pregnancy, when the total Tf concentrations were similar. Another argument for this conclusion was 
Distribution of transferrin subfractions ( $\%$ )

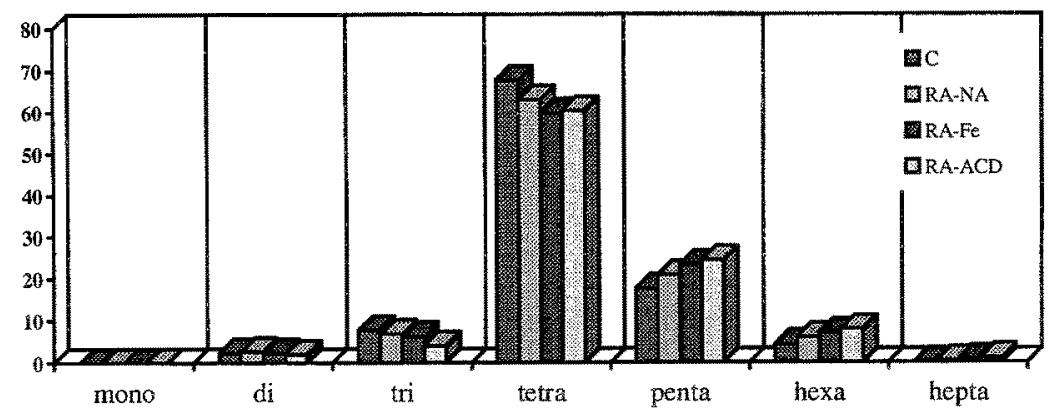

Transferrin subfraction concentration ( $\mathrm{mcmol} / \mathrm{)}$ )



Figure 7. Transferrin microheterogeneity in control females and females with RA divided in three groups: non-anaemic RA, RA accompanied by iron deficiency and RA complicated by anaemia of the chronic diseases. Top: proportional distribution of sialotransferrin fractions (\%). Bottom: corresponding concentrations $\left(\mu \mathrm{mol} \mathrm{I}^{-1}\right)$.

provided by the results from the RA group. In acute and chronic inflammatory conditions increased synthesis of acute phase proteins is invariably accompanied by changes in microheterogeneity and this suggests a linkage. On the other hand, $\mathrm{Tf}$ being a negative acute-phase protein [26] provides a model in which the effect of protein synthesis on N-glycosylation is dissociated from the effect of the acute phase response on glycosylation. The microheterogeneity pattern can change markedly even when protein synthesis is significantly depressed. Therefore, an association between the rate of Tf synthesis and the glycosylation pattern does not occur.

\section{Conclusion}

Accurate and reproducible description of $\operatorname{Tr}$ glycan microheterogeneity is provided by IF of iron saturated serum, and as such, provides a convenient tool for the study of $\mathrm{N}$-glycosylation. In a small scale this technique has been used to investigate certain clinical problems, and under these circumstances it provides valuable information. The lack of a more detailed understanding of the biochemistry of these changes will, however, probably limit their widespread use in the near future.

\section{References}

1. Loehr TH (ed.) (1989) Physical Bioinorganic Chemistry Series 19 5:239-370.

2. de Jong G, van Dijk JP and van Eijk HG (1990) Clin Chim Acta 190:1-46.

3. Yang F, Lum JB, McGill JR, Moore CM, Naylor SL, van Bragt PH, Baldwin WD, Bowman BH (1984) Proc Natl Acad Sci USA 81:2752-56.

4. Kornhuber B, Kühnl P (1986) In (Brinkmann B, Henningsem K eds) Advances in Forensic Haemogentics, vol. 1 pp. 119-22. Springer : Berlin, Heidelberg, New York.

5. Kamboh MI, Ferrell RE (1987) Hum Hered 37:65-81.

6. Spik G, Bayard B, Fournet B, Strecker G, Bouquelet S, Montreuil J (1977) FEBS Lett 50:296-99.

7. Hofman SW, Dwek RA, Fernandes DL, Rademacher TW (1982) FEBS Lett 150:503-6.

8. Dykes DD, Polesky HF (1984) CRC Crit Rev Clin Lab Sci 20:115-51.

9. Ritchie RF (1975) In (Putnam F, ed.) The Plasma Proteins, 2nd edn, vol 2, p. 375. Academic Press.

10. März L, Hatton MWC, Berry LR, Regoeczi E (1982) Can J Biochem 60:624-30.

11. Berger EG, Buddecke E, Kamerlingh JP, Kobata A, Paulson JC, Vliegenhart JFG (1982) Experentia 38:1129-258.

12. Schachter H (1984) Clin Biochem 17:3-14.

13. Kornfeld R, Kornfeld S (1985) Annu Rev Biochem 54:631-64. 
14. Roth J (1987) Biochim Biophys Acta 906:405-36.

15. Kobata A (1992) Eur J Biocham 209:483-501.

16. de Jong G, van Noort WL, Feelders RA, de Jeu-Jaspars CMH, van Eijk HG (1992) Clin Chim Acta 212:27-47.

17. Van Eijk HG, van Noort WL, Dubelaar ML, van der Heul C (1983) Clin Chim Acta 151:245-51.

18. Van Eijk HG, van Noort WL, de Jong G, Koster JF (1987) Clin Chim Acta 165:141-45.

19. Oberascher G, Arrer E (1986) Laryngol Rhinol Otol 8:161-64.

20. Jaeken J, van Eijk HG, van der Heul C, Corbeel L, Eeckels R, Eggermont E (1984) Clin Chim Acta 144:245-46.
21. Kristiansson B, Andersson M, Tonnby B, Hagberg B (1989) Arch Dis Child 64:71-76.

22. Ohno K, Yuasa I, Akaboshi S, Itoh M, Yoshida K, Ehara H, Ochiai Y, Takeshita K (1992) Brain Dev 14:30-35.

23. Jaeken J, Stibler H (1989) In Genetics of neuropsychiatric diseases, Wennergren International Symposium Series. (Wetterberg L, ed.) London: MacMillan Press, 51:69-80.

24. van Eijk HG, van Noort WL, de Jong G, Koster JF (1987) Clin Chim Acta 165:141-45.

25. de Jong G, van Eijk HG (1988) Electrophoresis 9:589-98.

26. Kushner I (1982) Ann NY Acad Sci 389:39-48. 